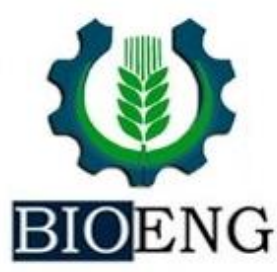

\title{
COMPARAÇÃO ENTRE ÍNDICES DE VEGETAÇÃO OBTIDOS POR IMAGENS AÉREAS COM VEÍCULO AÊREO NÃO TRIPULADO (VANT) E SATÉLITE
}

H. S. Sampaio ${ }^{1}$, V. Bourscheidt ${ }^{2}$, L. H. Sarracini ${ }^{3}$, L. A. C. Jorge ${ }^{4}$, G. M. Bettiol ${ }^{5}$, A. C. C. Bernardi ${ }^{6}$

${ }^{1}$ Tecnologia em Análise e Desenvolvimento de Sistemas, IFSP, São Carlos, SP, Brasil

${ }^{2}$ Departamento de Ciências Ambientais, UFSCar, São Carlos, SP, Brasil

${ }^{3}$ Aérials, São Carlos, SP, Brasil

${ }^{4}$ Embrapa Instrumentação, São Carlos, SP, Brasil

${ }^{5}$ Embrapa Cerrados, Planaltina, DF, Brasil

${ }^{6}$ Embrapa Pecuária Sudeste, Rodovia Washington Luiz, km 234, 13560-970, São Carlos, SP, Brasil.

Article history: Received 13 April 2020; Received in revised form 08 May 2020; Accepted 10 May 2020; Available online 30 June 2020.

\section{RESUMO}

O sensoriamento remoto (SR), amplamente utilizado em diferentes áreas do conhecimento, também pode ser aplicado para o manejo de sistemas agropecuários. Entre as diferentes técnicas aplicadas sobre os produtos de SR, pode se destacar o uso de índices de vegetação, que buscam relacionar variações do comportamento espectral medido através de imagens com diferentes parâmetros biofísicos das plantas. E a qualidade dos produtos resultantes depende diretamente da precisão das imagens utilizadas. Nesse sentido, este trabalho teve como objetivo comparar os índices de vegetação obtidos a partir de imagens de satélite e através de veículo aéreo não tripulado (VANT) em uma área de pastagem irrigada. Foram utilizadas imagens do satélite Landsat-8 e fotografias obtidas com câmeras acopladas em VANT (modelo convencional RGB e adaptada NGB, com bandas no visível e infravermelho próximo). Para as duas fontes de dados, foram calculados os seguintes índices: NDVI, o índice clássico no monitoramento da vegetação; o MPRI, que é correlato ao NDVI, porém se utiliza somente das bandas do espectro visível; o NDVI adaptado à câmeras digitais RGB e; o ENDVI, um aprimoramento do NDVI proposto para otimizar o uso de câmeras RGB. Os índices obtidos pelas duas fontes foram então correlacionados. A fim de tornar a comparação com imagens Landsat-8 mais adequada, os valores digitais das imagens de VANT foram convertidos para valores de reflectância. Os resultados mostraram uma correlação positiva intermediária dos índices NDVI e ENDVI obtidos a partir das imagens de satélite e VANT, que pode ter sido afetada tanto pela diferença de escala das imagens quanto pela variação temporal nas datas de aquisição de ambos os produtos. Evidenciou-se também a necessidade de calibração dos dados das imagens tomadas com VANTs, para garantir que a conversão para reflectância seja mais adequada. De modo geral, os índices estudados mostraram-se sensíveis para indicar as variações na área estudada e confirmaram que podem ser ferramentas de agricultura de precisão, auxiliando no planejamento do manejo das pastagens com a aplicação da Agricultura de Precisão.

Palavras-chave: Sensoriamento Remoto, VANT, Landsat-8, NDVI, MPRI, ENDVI.

\footnotetext{
*alberto.bernardi@embrapa.br
} 


\title{
COMPARISON BETWEEN VEGETATIVE INDEXES OBTAINED BY AERIAL IMAGES WITH UNMANNED AERIAL VEHICLES (UAV) AND SATELLITE
}

\begin{abstract}
Remote sensing (RS), which is widely employed in different areas, may be used as well for the management of agricultural systems. Among its different tools and applications, the use of vegetation indexes, which attempt to relate the variations of the measured spectral behavior with different biophysical parameters of the plants, may be one of the most important. And the quality of the resulting products will depend intrinsically on the precision of the images being used. On this way, this work aims to compare the vegetation indexes obtained from satellite images and with unmanned aerial vehicle (UAV) in an irrigated pasture area. The images were obtained from the Landsat- 8 satellite and with UAV (both conventional RGB and infrared adapted NBG cameras), and the following indices were calculated: NDVI, the classic index for monitoring vegetation; the MPRI, which is related to the NDVI, but uses only the bands of the visible spectrum; NDVI adapted to RGB digital cameras and; ENDVI, an enhancement of the NDVI proposed to optimize the use of RGB cameras. The values of the indices obtained by these images were further correlated. In order to make the comparison with Landsat- 8 images more appropriate, the digital values of UAV images were converted to reflectance values. The results of this study indicated an intermediate positive correlation of the NDVI and ENDVI indices obtained from satellite and UAV images, which could be influenced by the scale difference across the images, as well as by the temporal variation in the data acquisition from the different sources. The better calibration of the images taken with UAVs is also important to ensure the conversion to reflectance to be adequate. The studied indexes were sensitive to indicate the variations in the studied area and confirmed that they can be tools of precision agriculture, helping in the planning of pasture management with the application of Precision Agriculture.
\end{abstract}

Keywords: Remote Sensing, UAV, Landsat-8, NDVI, MPRI, ENDVI

\section{INTRODUÇÃO}

A Agricultura de Precisão (AP) é uma cadeia de conhecimentos, na qual máquinas, dispositivos, equipamentos e softwares são ferramentas para a coleta de dados, os quais devem ser organizados e interpretados, gerando informações para apoiar a gestão (INAMASU \& BERNARDI, 2014). Dessa forma, o emprego deste conjunto de tecnologias pode auxiliar os produtores na tomada de decisões gerenciais no manejo das culturas, levando em conta a variabilidade espacial e temporal da lavoura para obter máximo retorno econômico e reduzir $\mathrm{o}$ impacto ambiental (INAMASU et al., 2011). Geebers \& Adamchuk (2010) mostraram que a AP é um ciclo que se inicia na coleta dos dados, análises e interpretação dessas informações, geração das recomendações, aplicação no campo e avaliação dos resultados. A coleta de dados pode ser realizada com o uso de equipamentos ou sensores, ou a partir de imagens de sensoriamento remoto (SR), obtidas através de veículos aéreos (não) tripulados, ou em nível orbital através de satélite, por exemplo. $\mathrm{O}$ desenvolvimento de equipamentos e os métodos de SR têm possibilitado ampliar a capacidade de aquisição de dados em tempo real e em larga escala no campo. Por isso, o SR é considerado um dos pilares da AP, devido à capacidade de estimar produções e estimar índices de vegetação sem contato direto com as plantas (HATFIELD et al., 2008).

Os satélites e VANTs são ferramentas utilizadas em SR, cujo princípio das aplicações na agricultura baseia-se na interação de radiação eletromagnética com o solo ou a planta. 
A detecção remota na agricultura refere-se às medidas sem contato das radiações refletidas, ou emitidas em áreas agrícolas, podendo ser obtida por meio de satélites, veículos aéreos tripulados ou não tripulados, por sensores portáteis acoplados às máquinas agrícolas ou de forma manual (JORGE \& INAMASU, 2014; SHIRATSUCHI et al., 2014).

Imagens de satélite de média resolução tem sido utilizadas na agricultura através da cobertura sistemática em programas de monitoramento agrícola, sendo que as imagens Landsat têm sido usadas majoritariamente para previsões de safra (LILLESAND et al. 2004). De acordo com Albuquerque et al. (2017) as imagens de satélite comerciais com alta resolução espacial geralmente são comercializadas cobrindo áreas acima de 2.500 hectares, com alto custo de aquisição.

Já uso de VANT para a obtenção de imagens de grande resolução são uma alternativa às imagens de satélite (BERNI et al., 2009; AASEN et al., 2015), e tem despertado um interesse crescente para o monitoramento de sistemas agropecuários (LINHARES et al., 2013; JORGE \& INAMASU, 2014).

O cálculo dos índices de vegetação é realizado por combinações matemáticas dos valores da reflectância, sendo esses associados às características fisiográficas das plantas (SHIRATSUCHI et al., 2014, PEZZOPANE et al., 2019).

$O$ índice de vegetação por diferença normalizada (Normalized Difference Vegetation Index - NDVI), por ser uma ferramenta rápida e eficiente de detecção de variações na vegetação (ROUSE et al., 1973), é comumente usado para avaliar a sanidade, a biomassa e o teor de nutrientes das plantas (HATFIELD et al., 2008). Há vários relatos na literatura indicando a aplicabilidade deste índice (BERNARDI et al., 2014, 2017; PEZZOPANE et al., 2019).

A aplicação do NDVI depende da utilização de sensores para captura de imagens com comprimentos de onda no infravermelho próximo (NIR). Isso exige sensores mais caros que podem elevar os custos de aquisição e dificultar sua aplicação, sendo normalmente indicado para aplicação em áreas maiores.

O surgimento de sensores de alta resolução capazes de capturar imagens no espectro de comprimento visível e que podem ser transportados a bordo de VANTs domésticos, mais simples de manusear e pilotar, e de menor custo, têm estimulado os estudo de índices alternativos que utilizam as frequências de luz visível (RGB - Red, Green, Blue). Nesse contexto, o Modified Photochemical Reflectance Index (MPRI) foi proposto por Yang et al. (2008) e é baseado na diferença normalizada de duas bandas do espectro eletromagnético na região do verde e do vermelho, fazendo a substituição do infravermelho pelo verde na equação do NDVI. Com isso permite o seu uso com câmeras RGB (que detectam nos canais do vermelho, verde e azul), mais comuns e baratas para uso na aerofotogrametria (LINHARES et al., 2013). Barbosa et al (2019) empregaram este índice na avaliação do solo exposto e vegetado em área de cultivo de grama esmeralda (Zoysia japonica), alcançando resultados satisfatórios.

O NDVI foi adaptado para as imagens de VANT pelos próprios fabricantes de câmeras utilizadas nessas aeronaves, substituindo o sensor na faixa do vermelho (banda $\mathrm{R}$, ou red) por outro na faixa do NIR, e alterando a equação do NDVI para as bandas do NIR e do azul (banda B, ou blue). Isso porque, atualmente, a tecnologia digital das câmeras permite utilizar o canal vermelho da câmera como o canal que capta a reflexão da luz pela vegetação e o azul, por sua vez, como o canal que capta a absorção da luz (MAXMAX, 2015). A fórmula do NDVI para fotos aéreas obtidas com câmeras digitais pode ainda ser aprimorada com o Enhanced Normalized Difference Vegetaion Index (ENDVI), que é obtido pela soma da banda NIR com a banda verde (espectros refletidos por plantas 
saudáveis), e a banda azul, que é a de absorção, multiplicada por dois para compensar essa soma (MAXMAX, 2015). Susantoro et al. (2019) utilizaram com sucesso os índices NDVI e ENDVI para monitorar as etapas de crescimento da

\section{MATERIAL E MÉTODOS}

O estudo foi conduzido na Embrapa Pecuária Sudeste, em São Carlos, SP (21 ${ }^{\circ} 57^{\prime} 15 \mathrm{~S}$ e $47^{\circ} 50^{\prime} 53,5 \mathrm{~W}$; $856 \mathrm{~m}$ acima do nível do mar, Figura 1A), em área de solo Latossolo Vermelho-Amarelo distrófico, textura média (CALDERANO et al., 1998). O clima da região é tropical de altitude, com $1502 \mathrm{~mm}$ de precipitação pluvial anual e médias de temperatura mínima e máxima de $16,3^{\circ} \mathrm{C}$ (julho) e $23^{\circ} \mathrm{C}$ (fevereiro), respectivamente (ROLIM et al., 2007). A área de 8 ha de pastagem de Megathyrsus maximus cv. Mombaça (Figura 1B) vem sendo manejada no sistema intensivo desde 2005. As pastagens são manejadas em sistema rotacionado de pastejo com 3 e 4 dias de ocupação e 33 e 44 dias de descanso na época das águas e da seca, respectivamente. $\mathrm{O}$ pasto era adubado após a saída dos animais de cada piquete, com resíduo em torno de $40 \mathrm{~cm}$, na dosagem de $80 \mathrm{~kg} \mathrm{ha}^{-1}$ de nitrogênio por ciclo de pastejo, reduzida à metade no período da seca, e suspensa nos dois sistemas sem irrigação nesse período. A irrigação por aspersão foi realizada por meio de sistema autopropulsor de movimentação circular do tipo pivô central e o manejo da água (frequência e lâmina de irrigação). A pastagem de capim-mombaça era dividida com cerca eletrificada em 4 sistemas de 12 piquetes.

Para realizar a coleta das imagens aéreas, foram utilizados o VANT ISIS asa fixa da Hórus, Brasil e o VANT Echar asa fixa da XMobots, Brasil. O VANT ISIS foi equipado com uma câmera Canon S100 PowerShot de 12.1 megapixels, além de um GPS de navegação que registrava a geolocalização para cada imagem obtida durante o voo da aeronave. Com esse cultura da cana-de-açúcar.

Com base no exposto, este trabalho teve como objetivo comparar os índices de vegetação obtidos a partir de imagens de satélite e com VANT em uma área de pastagem irrigada.

equipamento, foi realizado um voo coletando-se imagens na faixa visível do espectro eletromagnético (RGB) no dia 16 de junho de 2015, com altura de aproximadamente 100 metros (960 metros acima do nível do mar) e por volta das 15 horas. As condições meteorológicas estavam favoráveis para fotos bem iluminadas.

O drone ou VANT Echar estava equipado com uma câmera de 24 MP e um GPS de navegação com a mesma função da nave ISIS. Com essa aeronave foram realizados dois voos no dia 5 de agosto de 2015, com altura de 300 metros (1.160 metros acima do nível do mar).

O primeiro voo ocorreu por volta das 11 horas, e capturou imagens nas bandas NGB (infravermelho próximo, verde e azul), e o segundo foi realizado por volta das 15 horas, capturando imagens RGB.

As imagens no espectro do visível e infravermelho do Landsat-8/OLI foram obtidas de banco de dados disponibilizado gratuitamente no site do United States Geological Survey (USGS http://earthexplorer.usgs.gov). Foram analisadas as imagens da área de estudo disponíveis no ano de 2015, e escolhidas duas delas que mais se aproximavam temporalmente dos levantamentos feitos com VANT: a primeira de 10 de junho de 2015 e a segunda para o dia 13 de outubro de 2015. Após o download do conjunto de bandas de cada imagem, essas foram corrigidas com o plugin Semi-Automatic Classification - SCP, para Quantum GIS. Este plugin aplica o método de correção atmosférica DOS1 (Dark Object Subtraction). O DOS1 é um método de correção atmosférica que utiliza os pixels escuros das imagens como referência para 
correção do espalhamento atmosférico, além de estimar a transmitância da atmosfera a partir do cosseno do ângulo zenital (SANCHES et al., 2011). Vale ressaltar que a diferença nas datas de aquisição das imagens por VANT e do satélite não é essencial nesse caso, tendo em vista o grande controle realizado em solo e que permite determinar as diferenças temporalmente.

A

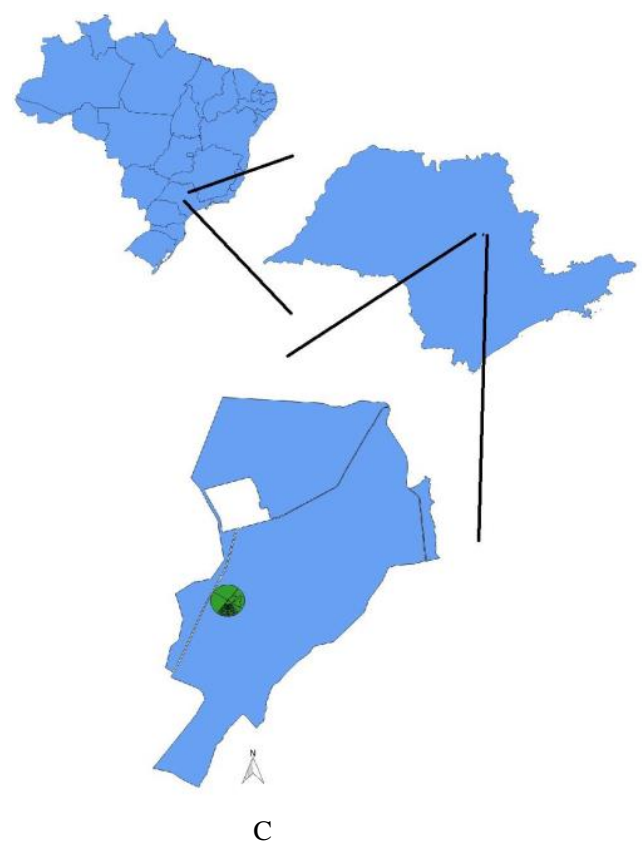

B



Figura 1. Localização da área de estudo, na Embrapa Pecuária Sudeste (A e B) e screenshot do processo de mosaicamento (C) no software Agisoft PhotoScan. São Carlos - SP.

O processamento fotogramétrico de imagens digitais e modelagem espacial 3D foi realizado com o software Agisoft Photoscan Pro (AgiSoft, 2014). Para as demais análises espaciais, foi utilizado o software de geoprocessamento QGIS, versões 2.8.1 e 2.12 (OSGEO, 2011).

Após a realização dos voos, as

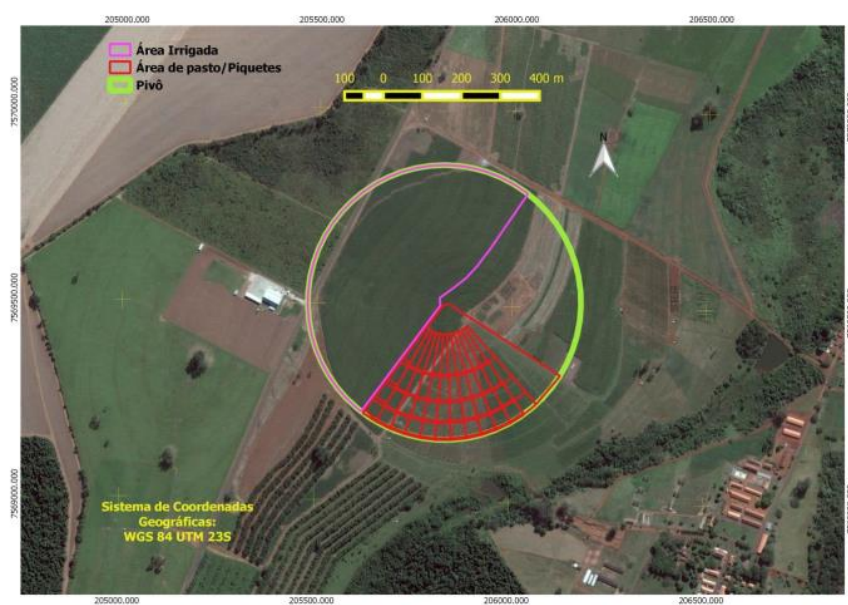


aeronaves durante o voo (movimento de inclinação provocada pelo vento, por exemplo) e as limitações de precisão do próprio GPS embarcado.

O processamento teve início transformando o conjunto de fotos em um mosaico, com o software PhotoScan (Figura 1C). Esse processamento é automático e utiliza dados de localização tridimensional e variação de luz nos pixels para encontrar semelhanças nas imagens e, dessa forma, agrupá-las (AGISOFT, 2014). $\mathrm{O}$ mosaico resultante possui como referência o sistema de coordenadas dos dados originais WGS 84.

Após a geração do mosaico, este foi exportado em formato Geotiff para pósprocessamento. Como os mosaicos gerados
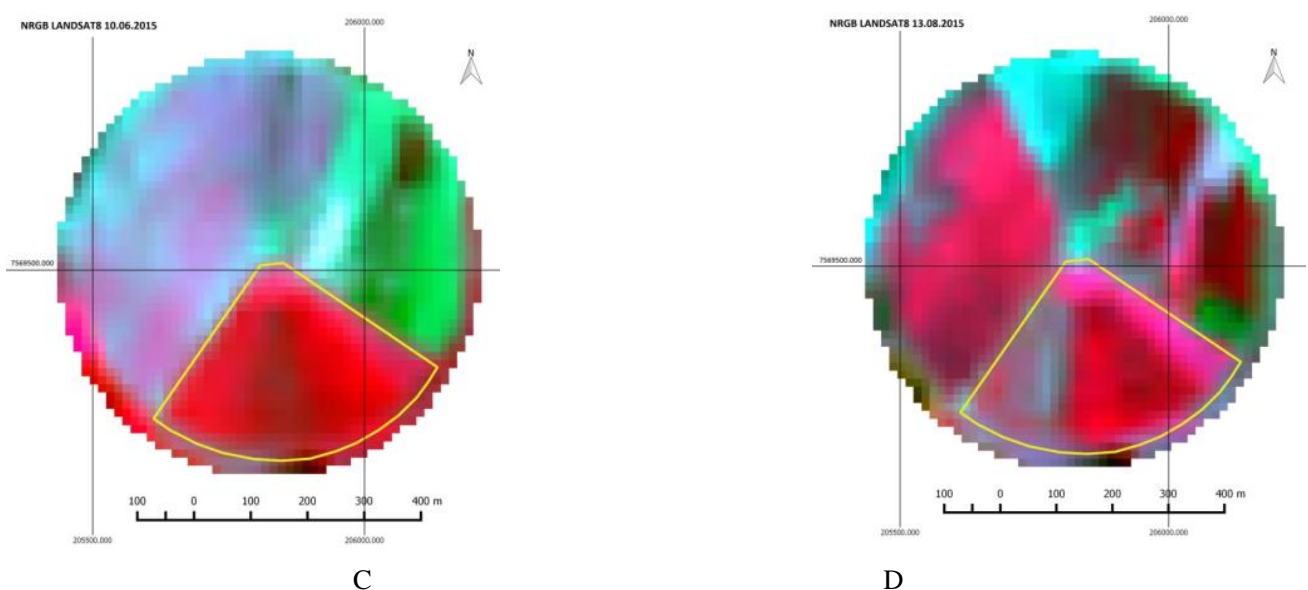

$\mathrm{D}$
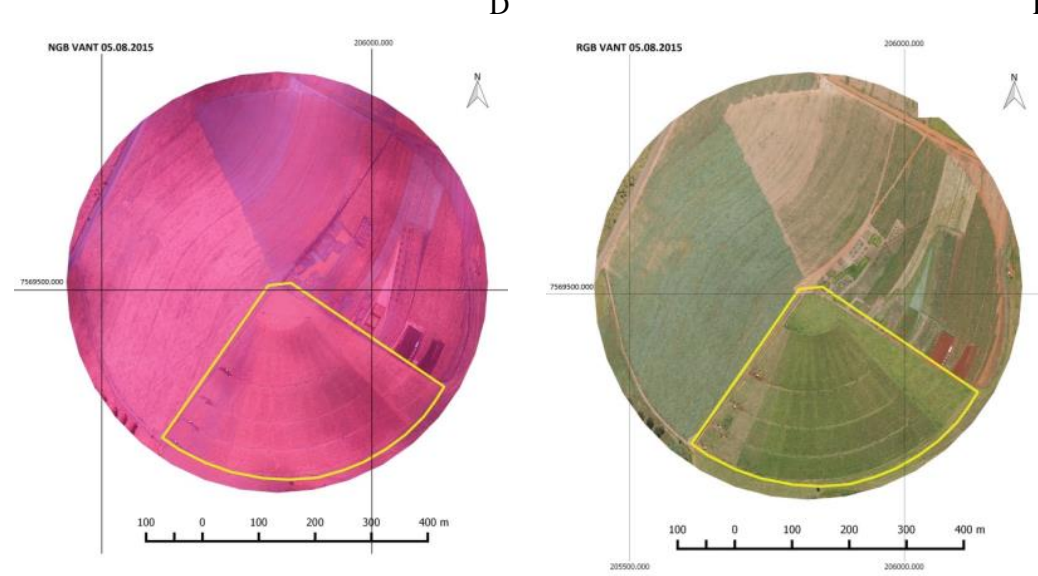

Figura 2. Imagens da área do pivô central obtidas a partir do Landsat-8 compostas nas bandas NRGB e fusionadas com a banda pancromática de resolução $15 \mathrm{~m}$ nas datas de amostragem de 10/06/15 (A) e 13/08/15 (B), e imagens obtidas com drones nas bandas RGB (C), NGB (D) e RGB (E) e em destaque a área de pastagem. São Carlos, SP. 
Os índices gerados a partir das imagens dos drones e das imagens do Landsat-8 foram normalizados para possibilitar uma comparação mais precisa. Para tal, fez-se uma adaptação da fórmula para conversão de valores de DN em reflectância usada em imagens Landsat-5, encontrada em Brito Neto et al. (2008) - a qual consiste da fusão das fórmulas da radiância e da reflectância monocromática discutidas por Markham \& Barker (1987), conforme a equação (1) a seguir:

$$
\rho_{\lambda}=\frac{\pi \cdot\left[\left(\frac{L_{\text {max }}-L_{\text {min }}}{D N_{\text {mix }}}\right) x(D N)+L_{\text {min }}\right]}{E_{\lambda} \cdot \cos (z) \cdot d_{r}}
$$

Onde: $\rho_{\lambda}=$ reflectância monocromática (para cada banda); $\mathrm{L}_{\text {máx }}$ e $\mathrm{L}_{\text {mín }}=$ são constantes da calibração do sensor utilizado; $\mathrm{DN}_{\text {máx }}=$ valor máximo que o $\mathrm{DN}$ pode alcançar; $\mathrm{DN}=$ número digital de cada pixel; $\mathrm{E}_{\lambda}=$ Irradiância solar média no topo da atmosfera $\left(\mathrm{mW} \mathrm{cm}{ }^{-2} \Omega^{-1} \mu \mathrm{m}^{-1}\right)$ para cada trecho do espectro eletromagnético (banda) analisado; $\mathrm{z}=$ ângulo solar no momento da aquisição da imagem; $d_{r}=$ inverso do quadrado da distância relativa Terra-Sol em unidades astronômicas.

Esta equação foi aplicada para cada banda de interesse e cada imagem obtida pelos VANTs. Esse cálculo foi realizado automaticamente pela Calculadora Raster do software Quantum GIS, gerando imagens Geotiff como arquivos de saída.

Embora os valores resultantes sejam apresentados em termos de reflectância, a equação foi usada principalmente para corrigir os efeitos da inclinação solar nos diferentes dias de tomada das imagens dos drones, na tentativa de equalizar essa variável, uma vez que as constantes de calibração específicas para os drones não foram determinadas (o valor adotado foi o mesmo que para o Landsat).

Em seguida, para aumentar a resolução das imagens Landsat, foi realizada a fusão por meio da banda pancromática para as bandas $\mathrm{N}, \mathrm{R}, \mathrm{G}$ e B (infravermelha, vermelha, verde e azul, ou bandas 5, 4, 3 e 2 do Landsat-8, respectivamente). Dessa forma, a resolução que originalmente era de 30 metros por pixel, aumentou para 15 metros por pixel (Figura 2).

O cálculo do NDVI (Figura 3A) foi feito pela diferença de refletâncias entre a banda do infravermelho próximo (NIR) e a do vermelho (VIS), normalizada pela divisão da soma das bandas de NIR e VIS (Rouse et al., 1973), expressado pela Equação 2:

$$
\mathrm{NDVI}=\frac{N I R_{760}-V I S_{670}}{N I R_{760}+V I S_{670}}
$$

Onde:

$\mathrm{NIR}_{760}$ e VIS $670=$ refletâncias das bandas do infravermelho próximo e vermelho, respectivamente.

O MPRI (Figura 3 B, C, D e E) foi calculado conforme a equação 3 a seguir proposta por Yang et al. (2008):

$$
\text { MPRI }=\frac{G-R}{G+R}
$$

Onde:

$\mathrm{G}$ e $\mathrm{R}$ = refletâncias das bandas do verde e vermelho, respectivamente.

O índice NDVI adaptado (Figura 3 F) para as câmeras digitais RGB foi calculado de acordo com Maxmax (2015) pela equação (4):

$$
\mathrm{NDVI}=\frac{R_{I V P}-R \quad B}{R_{I V P}+R_{B}}
$$

Onde:

$\mathrm{R}_{\mathrm{IVP}}=$ reflectância no infravermelho próximo; e $\mathrm{R}_{\mathrm{B}}$ - reflectância no azul. 
Já o Enhanced Normalized Difference Vegetaion Index (ENDVI, Figura $3 \mathrm{G}$ ) foi calculado de acordo com Maxmax (2015) pela equação (5):

$$
\text { ENDVI }=\frac{\left(R_{I V P}+R_{G}\right)-2\left(\begin{array}{ll}
R & B
\end{array}\right)}{\left(R_{I V P}+R_{G}\right)+2\left(\begin{array}{ll}
R & B
\end{array}\right)}
$$

Onde:

$\mathrm{R}_{\mathrm{IVP}}=$ reflectância no infravermelho próximo; $\mathrm{R}_{\mathrm{G}}=$ reflectância no verde; e $\mathrm{R}_{\mathrm{B}}$ $=$ reflectância no azul.

Os mapas com os recortes de cada índice foram gerados com o software QGIS (OSGEO, 2011).
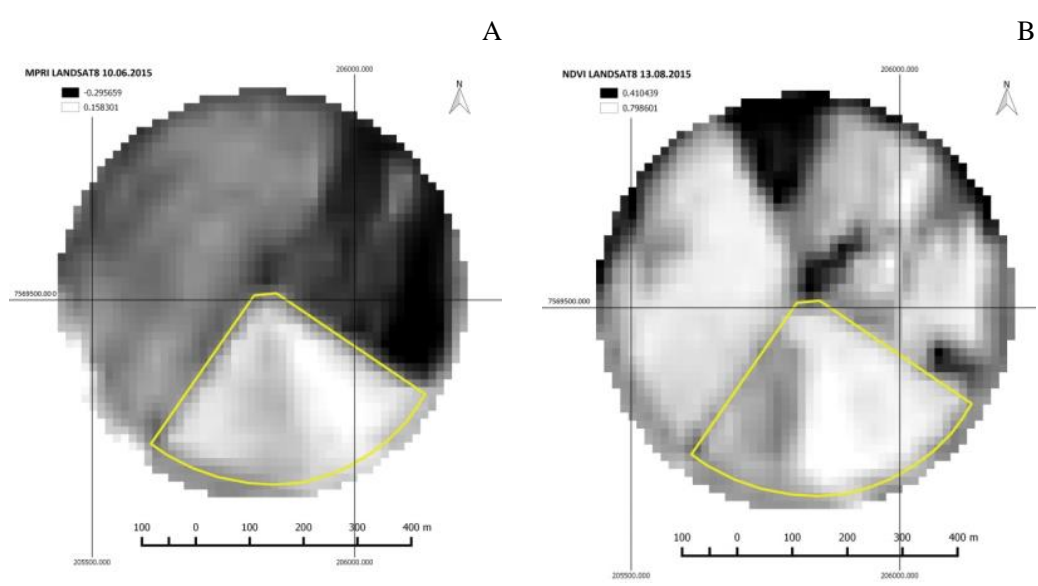

B

C

D
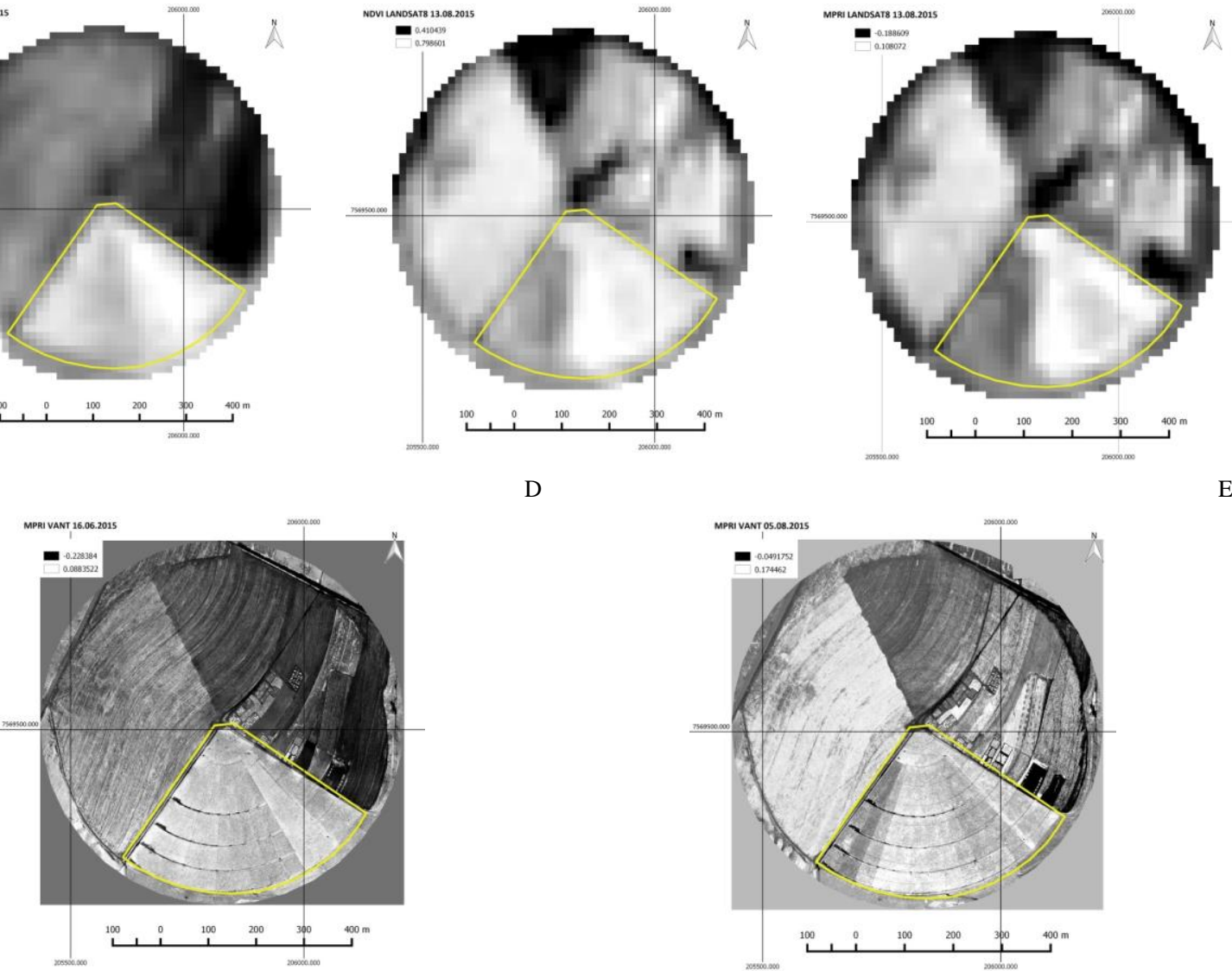

F
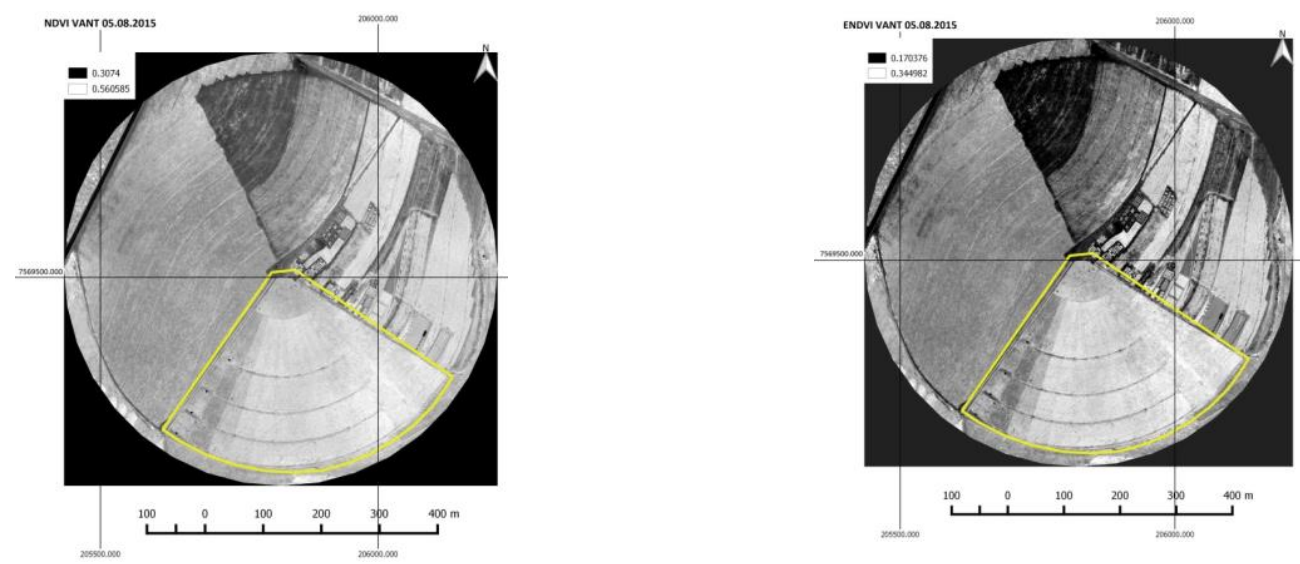

Figura 3. Índices MPRI (A e C) e NDVI (C) a partir das imagens Landsat-8, e MPRI para as imagens RGB (D e E) e NDVI adaptado (F) e ENDVI (G) calculados para a imagem aérea NGB adquiridas com drones. 


\section{RESULTADOS E DISCUSSÃO}

Os índices para todas as imagens obtidas a partir do Landsat-8 e VANTs (Figura 2) foram calculados. Para a análise, destacou-se a área de pasto na porção sudeste do pivô (Figura 3). Esta área foi escolhida para o estudo pois possui maior variabilidade para comparação, uma vez que é vegetada o ano todo, e o pasto é manejado de forma rotacionada, sendo que em uma mesma imagem é possível detectar-se diferenças na biomassa da pastagem.

Com base nos resultados apresentados nas Figuras 3A, 3D e 3E, pode-se inferir que o MPRI é um dos índices mais adequados para avaliar as diferenças da vegetação e a cobertura do solo, uma vez que proporcionou a adequada distinção visual entre essas variáveis. Estas diferenças visuais estão presentes, mesmo que a amplitude de variação dos resultados não seja grande $(0,2)$. Observando as imagens, destacam-se as áreas que estão sendo pastejadas (áreas à esquerda), e aqueles piquetes que entrarão na rotação (à direita) por já terem rebrotado, apresentando maior biomassa. Estudos prévios realizados por Barbosa et al. (2019) com grama esmeralda ( $Z$. japonica) também obtiveram resultados positivos com este índice. Porém os autores alertam que o MPRI pode ser afetado pelo sombreamento das nuvens, interferindo na correta interpretação da imagem.

O NDVI depende do contraste espectral entre as bandas vermelho e infravermelho próximo, e está relacionado à quantidade de biomassa e ao teor e conteúdo de pigmentos vegetais (NUMATA et al., 2007; HATFIELD et al., 2008). Dessa forma, pode se verificar nos mapas (Figura 3B) que os aumentos do índice NDVI indicam maior produção (áreas mais escuras) da parte aérea, com valores variando de 0,41 a 0,8 . Esta afirmação é corroborada pelo trabalho de Pezzopane et al. (2019), que estimaram a biomassa de capim Piatã (Urochloa brizantha) em diferentes condições de cultivo e obtiveram coeficientes de determinação entre 0,65 e 0,93 relacionando o NDVI à produção da pastagem.

A comparação da Figura 2E com a Figura 3G indica que, embora as variações nos estágios de utilização da pastagem possam ser observadas na imagem original (Figura 2E), sua variação se torna muito mais evidente ao utilizar o ENDVI. O lado esquerdo apresenta maiores valores de ENDVI, indicando maior biomassa, enquanto que, a medida que se caminha para a direita os valores diminuem. Isso é explicado pela diferença dos estágios de pastejos. Cada faixa (ou subdivisão) é ocupado pelos animais por três dias, que na sequencia utilizam a próxima faixa. Dessa forma, o ENDVI confirma estas utilizações, nas faixas da direita o pasto está mais crescido, com mais folhas, e mais verde, enquanto que as faixas da esquerda indicam que ele já foi consumido. Susantoro et al. (2019) também mostraram que o ENDVI foi útil para diferenciar as fases de crescimento, porém para o caso da cultura da cana-de-açúcar.

Já o NDVI adaptado (Figura 3F) não possibilitou uma grande diferenciação das áreas de utilização de pastagem, uma vez que houve poucas diferenças entre os valores (de 0,31 a 0,56) indicando um menor potencial de utilização deste índice de vegetação nas condições testadas. De acordo com Jorge \& Inamasu (2014) as imagens no espectro visível $(0,4$ a $0,7 \mu \mathrm{m})$, funcionam como o "olho do agricultor" sobre a cultura, uma vez que possibilitam obtenção de imagens que auxiliam na detecção de falhas de plantio, alterações no dossel. No entanto, algumas variáveis como a detecção de estresse nutricional, indicadores fisiológicos e estrutura do dossel são mais bem verificados com imagens no infravermelho, ou através de combinações em índices, como é o caso do NDVI.

Após a análise qualitativa, procedeu- 
se $\mathrm{o}$ recorte das áreas de interesse destacando-se a pastagem, para a comparação normalizada dos valores dos índices obtidos a partir das imagens do satélite e do drone. Dessa forma, nas imagens adquiridas com os drones, foi obtida uma média dos pixels equivalentes à área de um pixel da imagem Landsat-8, que tem a resolução menor. Com essa transformação, foi possível fazer um teste de correlação de Pearson (r) entre os dados normalizados. As imagens das comparações dos índices com escala original, bem como os valores de correlação obtidos, podem ser conferidas na Tabela 1. Observando as correlações, constata-se que as variáveis MPRIVANT e MPRI Landsat8 (nas datas de 16 e 10/jun) apresentaram correlações positivas de valor intermediário $(0,61)$. Já os resultados obtidos para as variáveis MPRIvant e MPRI Landsat8 (nas datas de 05 e 13/ago) indicam que estes estão fracamente correlacionadas, pois o valor de $\mathrm{r}$ obtido foi de 0,39. A comparação entre o NDVIVANT e ENDVIVANT com o NDVI Landsat8 (nas datas de 05 e 13/ago) também indicaram valores positivos medianamente relacionados $(0,597$, e 0,586 , respectivamente).

Já a comparação de índices mais distintos, como entre o NDVIVANT e ENDVIVANT com o MPRI Landsat8 (nas datas de 05 e 13/ago) também mostrou valores positivos de 0,599 e 0,598 , considerados intermediários.

Tabela 1. Coeficiente de correlação de Pearson (r) dos índices obtidos a partir das imagens do satélite Landsat-8 e das imagens com VANT nas datas estudadas.

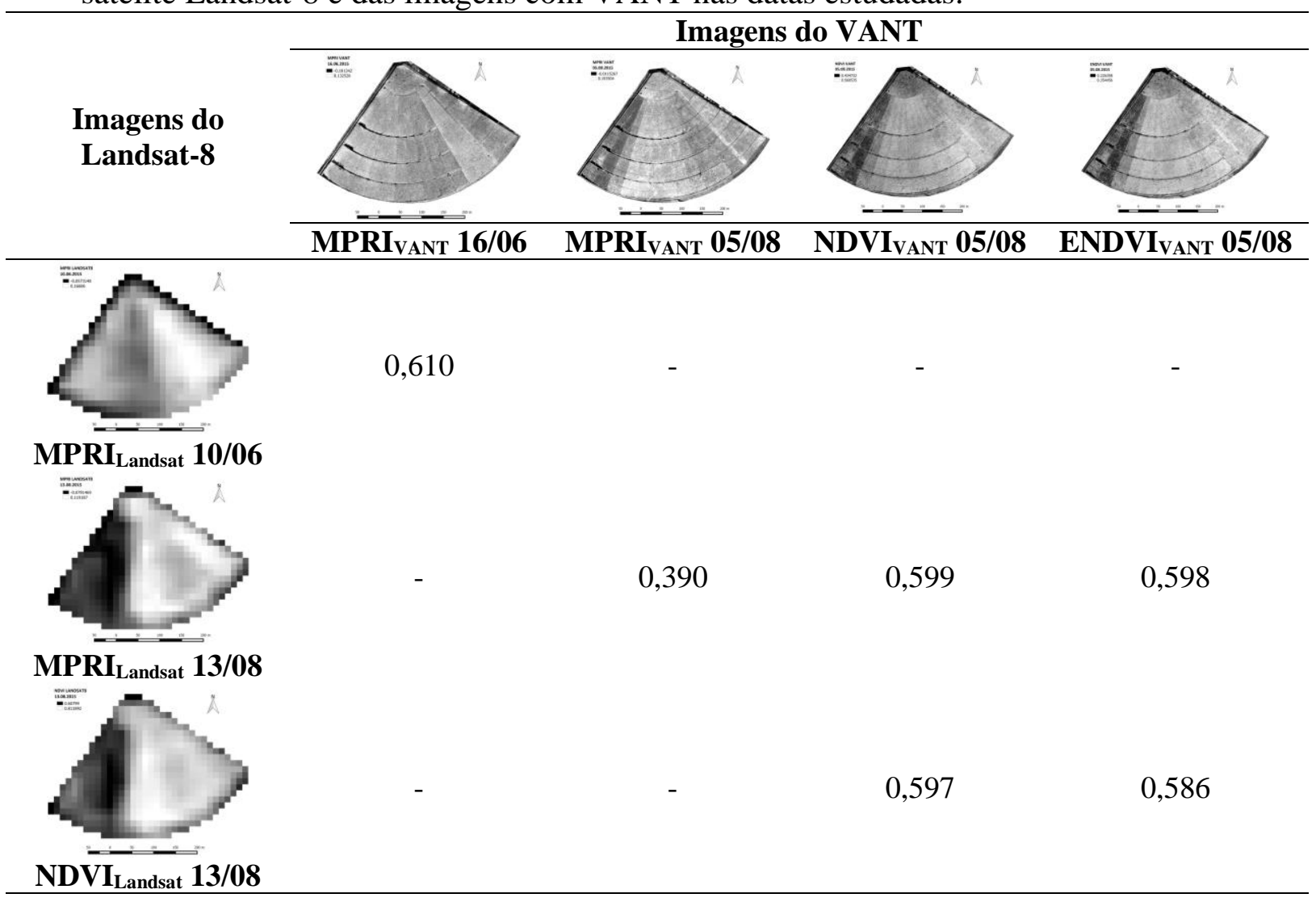

É importante ressaltar que essa análise negligencia a variação temporal na aquisição das imagens, que pode ter alguma influência nos valores obtidos. Outro ponto que deve ser ressaltado refere- se a falta de pontos de controle em campo para ortorretificação das imagens dos VANTs, que também pode ter interferido na qualidade dos dados, uma vez que possivelmente houve alguma distorção da 
imagem em relação ao relevo real. Além disso, há que se considerar à diferença de escala e a falta de calibração dos dados obtidos nas imagens aéreas. Jorge \& Inamasu (2014), Aasen et al. (2015) e Albuquerque et al (2017) já haviam ilustrado a diferença de resolução das imagens com os VANTs comparada com imagens de satélites convencionais. Além disso, Linhares et al. (2013) e Aasen et al. (2015) já indicavam que o emprego de VANTs era uma tecnologia com crescente destaque, principalmente para a coleta de imagens que visam subsidiar $\mathrm{o}$ mapeamento em grandes escalas.

A ineficiência ou invalidade da aplicação dos índices de vegetação às imagens de VANTs é pouco provável, uma vez que é possível perceber as áreas com maior biomassa diferenciadas das com menor biomassa nos mapas. Além disso, a relação dos índices NDVI com a produção de biomassa já foi demonstrada em diversos outros estudos, como Bernardi et al. (2014, 2017), e Pezzopane et al. (2019).

Jorge \& Inamasu (2014) ainda destacam que, devido à maior resolução das imagens de VANT, essas possibilitam a observação de diferenças detalhadas da produção de biomassa, e permitem por exemplo, acompanhar o crescimento da planta no campo. Outra vantagem, segundo os autores, está na possibilidade de variação das resoluções das imagens de centímetros a metros, dependendo da altura de voo e lente utilizada. Albuquerque et al

\section{CONCLUSÕES}

Os resultados deste estudo indicaram que o sensoriamento remoto foi útil para o mapeamento da variabilidade espacial da pastagem.

Há a necessidade de calibração dos dados das imagens tomadas com VANTs, para que a conversão para reflectância seja adequada, permitindo uma comparação mais efetiva.

A escala das imagens pode diferenciar os resultados.
(2017) confirmaram que com os VANTs é possível sobrevoar somente a área de interesse, gerando imagens de maior resolução espacial. E como destacaram Berni et al. (2009), os VANTs permitem a coleta de imagens de difícil acesso, e geram imagens de sem interferência de nuvens.

Também é interessante mencionar que é possível fazer adaptações dos índices ou mesmo a utilização de índices distintos dos usados neste estudo, como os apresentados por Shiratsuchi et al. (2014), por exemplo. Além disso, a verificação da aplicabilidade destes índices nas novas escalas de SR podem ser interessantes de serem estudadas (ou mesmo necessárias).

Os esforços futuros para o estudo na área de pastagem envolveriam correlação dos índices de vegetação com outros fatores, como irrigação, conteúdo de nutrientes do solo, estado nutricional das plantas, qualidade e produção de biomassa, seguindo o exemplo do trabalho de Pezzopane et al. (2019). Outro ponto seria a coleta de imagens em diferentes épocas do ano, especialmente no verão, pois é período de maioro crescimento de biomassa da pastagem avaliada.

E, para uma melhor compreensão da aplicabilidade das imagens de SR para a $\mathrm{AP}$, são necessários estudos de validação, de comparação tanto com as formas de medidas clássicas de SR quanto de comparação com metodologias de medidas em campo.

Houve correlação positiva intermediária dos índices NDVI e ENDVI obtidos a partir das imagens de satélite e VANT.

Os índices estudados mostraram-se sensíveis para indicar as variações na área estudada e confirmaram que podem ser ferramentas de agricultura de precisão, auxiliando no planejamento do manejo das pastagens com a aplicação da Agricultura de Precisão. 


\section{REFERÊNCIAS}

AASEN, H.; BURKART, A.; BOLTEN, A.; BARETH, G. Generating 3D hyperspectral information with lightweight UAV snapshot cameras for vegetation monitoring: From camera calibration to quality assurance. ISPRS Journal of Photogrammetry and Remote Sensing, v. 108, p. 245-259, 2015.

AGISOFT LLC (2014) - Agisoft Photoscan Pro. St. Petersburg, Russia. Disponível em: http://www.agisoft.com

ALBUQUERQUE, R. W. DE., COSTA, M. O., FERREIRA, M. E., JORGE, L. A. C., SARRACINI, L. H., ROSA, E. D. Uso do índice MPRI na avaliação de processos de Restauração Florestal (RF) utilizando sensor RGB a bordo de VANT quadricóptero. In: SEMINÁRIO DE ATUALIZAÇÃO EM SENSORIAMENTO REMOTO E SISTEMAS DE INFORMAÇÕES GEOGRÁFICAS APLICADOS À ENGENHARIA FLORESTAL, 18., 2017, Santos. Proceedings... São José dos Campos: INPE, 2017. p. 4795-4802 Disponível em: $<$ https://proceedings.science/proceedings/5 9/_papers/60224/download/abstract_file1>

BARBOSA, B. D. S.; FERRAZ, G. A. S.; GONÇALVES, L. M.; MARIN, D. B.; MACIEL, D. T.; FERRAZ, P. F. P.;

ROSSI, G. RGB vegetation indices applied to grass monitoring: a qualitative analysis. Agronomy Research, v.17, p.349-357, 2019. https://doi.org/10.15159/AR.19.119

BERNARDI, A. C. C.; GREGO, C. R.; ANDRADE, R. G.; RABELLO, L. M.; INAMASU, R. Y. Spatial variability of vegetation index and soil properties in an integrated crop-livestock system. Revista Brasileira de Engenharia Agrícola e Ambiental, v.21, n.8, p.513-518, 2017.

BERNARDI, A. C. C.; RABELLO, L. M.; INAMASU, R. Y.; GREGO, C. R.;
ANDRADE, R. G. Variabilidade espacial de parâmetros físico-químicas do solo e biofísicos de superfície em cultivo do sorgo. Revista Brasileira de Engenharia Agrícola e Ambiental, v. 18, p. 623-630, 2014.

BERNI, J. A. J.; ZARCO-TEJADA, P. J.; SUAREZ, L.; FERERES, E. Thermal and Narrowband Multispectral Remote Sensing for Vegetation Monitoring from an Unmanned Aerial Vehicle. IEEE Transactions on Geoscience and Remote Sensing, v. 47, n. 3, p. 722-738, 2009.

BRITO NETO, R. T.; BARROS FILHO, M. B. B.; LOPES, H. L.; PACHECO, A. P. Determinação de valores físicos de imagens TM/Landsat- 5 utilizando a linguagem legal para obter índices de vegetação. In: SIMPÓSIO BRASILEIRO DE CIÊNCIAS GEODÉSICAS E TECNOLOGIAS DA GEOINFORMAÇÃO, 2, Recife, 2008. Anais. Recife, UFPE.

CALDERANO FILHO, B.; SANTOS, H. G.; FONSECA, O. O. M.; SANTOS, R. D.; PRIMAVESI, O.; PRIMAVESI, A. C. Os solos da Fazenda Canchim, Centro de Pesquisa de Pecuária do Sudeste, São Carlos, SP: levantamento semidetalhado, propriedades e potenciais. Rio de Janeiro: EMBRAPA-CNPS; São Carlos, SP: EMBRAPA-CPPSE, 1998.95 p. il. (EMBRAPA-CNPS. Boletim de pesquisa, 7; EMBRAPA-CPPSE. Boletim de pesquisa, 2).

HATFIELD, J. L.; GITELSON, A. A.; SCHEPERS, J. S.; WALTHALL, C. L. Application of spectral remote sensing for agronomic decisions. Agronomy Journal, v.100, p.117-131, 2008.

INAMASU, R. Y.; BERNARDI, A. C. C.; VAZ, C. M. P.; NAIME, J. M.; QUEIROS, L. R.; RESENDE, A. V.; VILELA, M. de F.; JORGE, L. A. C.; BASSOI, L. H.; 
PEREZ, N. B.; FRAGALLE, E. P. Agricultura de precisão para a sustentabilidade de sistemas produtivos do agronegócio brasileiro. In: INAMASU, R. Y.; NAIME, J. M.; RESENDE, A. V.; BASSOI, L. H.; BERNARDI, A. C. C. (Ed.). Agricultura de precisão: um novo olhar. São Carlos: Embrapa Instrumentação, 2011. p. 14-26.

INAMASU, R. Y.; BERNARDI, A. C. C. Agricultura de precisão. In: BERNARDI, A. C. C.; NAIME, J. M.; RESENDE, A. V.; BASSOI, L. H.; INAMASU, R. Y. (Ed.). Agricultura de precisão: resultados de um novo olhar. Brasília, DF: Embrapa, 2014. p. 21-33.

JORGE, L. A. C.; INAMASU, R. Y. Uso de veículos aéreos não tripulados (VANT) em agricultura de precisão. In:

BERNARDI, A. C. C.; NAIME, J. M.; RESENDE, A. V.; BASSOI, L. H.; INAMASU, R. Y. (Ed.). Agricultura de precisão: resultados de um novo olhar. Brasília, DF: Embrapa, 2014. p. 109-134.

LILLESAND, T. M.; KIEFER, R. W.; CHIPMAN, J. W. Remote Sensing and Image Interpretation, $5^{\text {a }} \mathrm{Ed}$. New York: John Wiley \& Sons, 2004. 763p.

LINHARES, M. M. A.; ROCHA, N. C. C.; AMARAL, B. A. S. Análise do índice MPRI como indicador vegetativo através da correlação do mesmo com o índice NDVI. In: SIMPÓSIO BRASILEIRO DE SENSORIAMENTO REMOTO, 16. (SBSR), 2013, Foz do Iguaçu. Anais... São José dos Campos: INPE, 2013. p. 82548260. Disponível em: <http://www.dsr.inpe.br/sbsr2013/files/p11 90.pdf $>$.

MARKHAM, B. L.; BARKER, J. L. Radiometric properties of US processed Landsat MSS data. Remote Sensing of Environment, v. 22, n. 1, p. 39-71, 1987. MAXMAX. Enhanced Normalized Difference Vegetation Index (ENDVI). Disponvível em: https://www.maxmax.com/maincamerapag e/remote-sensing/enhanced-normalizeddifference-vegetation-index. Acesso em: 27 mar 2015.

NUMATA, I.; ROBERTS, D. A.; CHADWICK, O. A.; SCHIMEL, J.; SAMPAIO, F. R.; LEONIDAS, F. C.; SOARES, J. V. Characterization of pasture biophysical properties and the impact of grazing intensity using remotely sensed data. Remote Sensing of Environment, v.109: 314-327, 2007.

OSGEO - Open Source Geospatial Foundation. 2011. Quantum GIS (QGIS). Open Source Geographic Information System (GIS). Version 1.6.0. Disponível em: <http://qgis.org/>

PEZZOPANE, J. R. M.; BERNARDI, A. C. C.; BOSI, C.; CRIPPA, P. H.; SANTOS, P. M.; NARDACHIONE, E. C. Assessment of Piatã palisadegrass forage mass in integrated livestock production systems using a proximal canopy reflectance sensor. European Journal of Agronomy, v. 103, p. 130-139, 2019.

ROLIM, G. D. S.; CAMARGO, M. B. P. D.; LANIA, D. G.; MORAES, J. F. L. D. Climatic classification of Köppen and Thornthwaite sistems and their applicability in the determination of agroclimatic zonning for the state of São Paulo, Brazil. Bragantia, v.66(4): 711720, 2007.

ROUSE, J.W.; HAAS, R.H.; SCHELL, J.A.; DEERING, D.W. Monitoring vegetation systems in the Great Plains with ERTS. In: EARTH RESOURCES TECHNOLOGY SATELLITE SYMPOSIUM, 1., 1973, Washington. Proceedings. Washington: NASA, 1973. v.1, p.309-317.

SANCHES, I.; ANDRADE, R. G.; QUARTAROLI, C. F.; RODRIGUES, C. A. G. Análise comparativa de três métodos de correção atmosférica de imagens 
Landsat 5 - TM para obtenção de reflectância de superfície e NDVI. In: Simpósio Brasileiro de Sensoriamento Remoto (SBSR), 15., 2011, Curitiba.

Anais. São José dos Campos, SP. INPE, 2005. p 7564-7571. Acesso em 22 de agosto de 2015.

SHIRATSUCHI, L. S.; BRANDAO, Z. N.; VICENTE, L. E.; VICTORIA, D. C.; DUCATI, J. R.; OLIVEIRA, R. P.; VILELA, M. F. Sensoriamento Remoto: conceitos básicos e aplicações na Agricultura de Precisão. In: BERNARDI, A. C. C.; NAIME, J. M.; RESENDE, A. V.; BASSOI, L. H.; INAMASU, R. Y. (Ed.). Agricultura de precisão: resultados de um novo olhar. Brasília, DF: Embrapa, 2014. p. 58-73.

SUSANTORO, T. M.; WIKANTIKA, K.; HARTO, A. B.; SUWARDI. D.

Monitoring sugarcane growth phases based on satellite image analysis (a case study in Indramayu and its Surrounding, West Java, Indonesia). HAYATI Journal of

Biosciences, v.26, p.117-128, 2019.

DOI:10.4308/hjb.26.3.117.

YANG, Z.; WILLIS, P.; MUELLER, R. Impact of Band-Ratio Enhanced AWIFS Image to Crop Classification Accuracy. In: Pecora - The Future of Land Imaging... Going Operational, Denver, Colorado, USA. Proceedings. Maryland: (ASPRS), 2008. 